Product

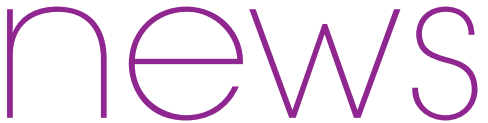

Product news is provided as a service to readers using text and images from

the manufacturer, supplier or distributor and does not imply endorsement

by BDJ Team. Normal and prudent research should be exercised before

purchase or use of any product mentioned.

\section{SPECIALISED KITS FOR COSMETIC TREATMENTS}

Tandex provides quality aftercare kits for maintaining good levels of oral hygiene during and after cosmetic treatments.

Now more than ever your patients have access to unprecedented levels of cosmetic dentistry and it is important that you are ready to provide them with the equipment they need to thoroughly look after their teeth throughout these treatments.

Tandex offers both orthodontic and implant kits that have all the necessary tools and adjuncts to ensure your patients can complv with anv specific aftercare instruction - from gentle brushes for sensitive areas of the gingiva to interproximal brushes to ensure a thorough clean.

The kits include detailed user guides that comprehensively instruct patients on optimum cleaning protocols. Produced in cooperation with dentists and hygienists the in-depth information allows patients to use the products as they were intended, for easily achievable and excellent results.

Ensure you are ready to guide your patients to uphold strong standards of oral hvgiene during and after cosmetic treatments by offering Tandex's range of specialist kits. For more information on Tandex's range of products call +454738 1001, email tandex@ tandex.dk or visit www. tandex.dk.

\title{
DRIVING DENTAL INNOVATION TO YOUR PRACTICE
}

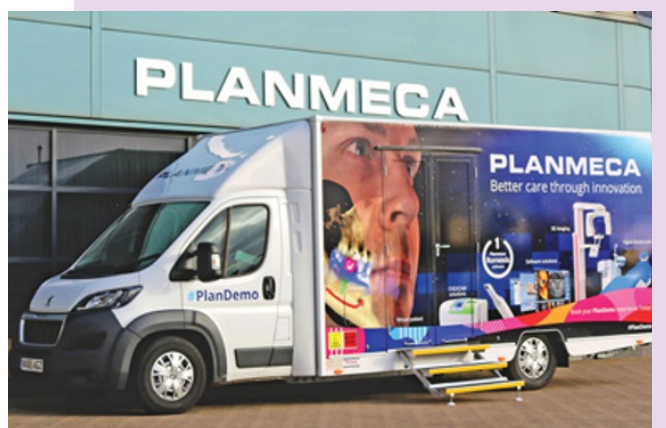

Planmeca has always driven dental innovation and now we are driving innovation right to your door. Our state of the art mobile experience centre: PlanDemo, is ready to visit your practice to showcase the latest range of dental innovations that make up the award

winning Planmeca product portfolio. What's more, every practice visit qualifies for verifiable CPD.

You can be sure that you and your dental team will have every opportunity to discover, learn and connect to a world of digital dentistry; from the high-tech Compact i Touch dental unit featuring class leading integration and automatic infection control, to a fully functioning ProMax 3D, ProX intraoral scanner and our full chairside CAD/CAM solution; Planmeca FIT.

Interested in a visit from PlanDemo? Call 0500500686 or alternatively visit www. plandemo.co. uk to connect to a world of digital dentistry.

\section{THE ELITE OF SELF-ADHESIVE RESIN CEMENTS}

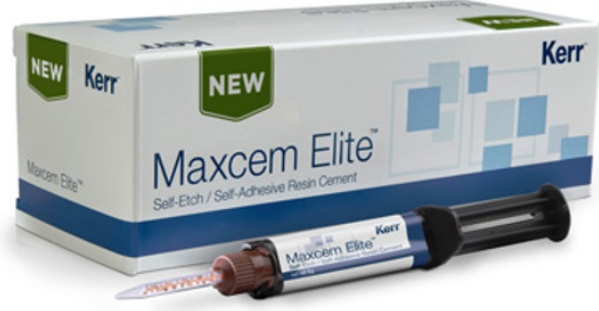

Kerr's Maxcem Elite came out on top

in recent research comparing the

bond strength of various self-adhesive

dental cements to human dentine and

enamel. Following a 2015 laboratory

evaluation, Brown and colleagues

concluded that new and improved

Maxcem Elite, a self-etch, self-adhesive

resin cement for indirect restorations,

showed superior adhesive bonding

results across all tested products in

regards to shear bond strength:

In addition to its superior bond

strength, Maxcem Elite offers:

- Easy handling

- Simple procedures

- Universal application

The material's optimised resin matrix and filler systems also improve wettability, allowing for immediate light curing and better shelf-life stability.

Also worthy of note is that its

proprietary redox initiator system

eliminates the inherent discolouration of benzoyl peroxide/tertiary amine initiator systems for a more aesthetic restoration.

For further information, call 01733 892292, email kerruk@kerrhawe.com or visit www.kerrdental.co.uk. 